\title{
Plasmonic biodegradable gold nanoclusters with high NIR-absorbance for biomedical imaging
}

\author{
Robert Stover*1, Avinash Murthy ${ }^{2}$, Sai Gourisankar ${ }^{2}$, Golay $\mathrm{Nie}^{2}$, Miguel Martinez ${ }^{2}$, Thomas \\ Truskett $^{2}$, Konstantin Sokolov ${ }^{3,4}$, Keith Johnston ${ }^{1,2}$ \\ 1. Texas Materials Institute, University of Texas at Austin, Austin, TX 78712 \\ 2. Department of Chemical Engineering, University of Texas at Austin, Austin, TX 78712 \\ 3. Department of Biomedical Engineering, University of Texas at Austin, Austin, TX 78712 \\ 4. Department of Imaging Physics, The UT M.D. Anderson Cancer Center, Houston, TX 77030
}

\begin{abstract}
Gold plasmonic nanoparticles are receiving attention for a variety of types of NIR optical biomedical imaging including photoacoustic imaging. Herein we present a novel method to assemble equilibrium gold nanoclusters from 5 $\mathrm{nm}$ primary gold nanospheres, which exhibit high near-infrared (NIR) absorbance and subsequently fully dissociate back to primary particles, which has the potential to enable renal clearance. The nanoparticle assembly is manipulated via controlling colloidal interactions, specifically electrostatic repulsion and depletion attraction. The charge on the primary $\sim 5 \mathrm{~nm}$ gold nanospheres is tailored via place exchange reactions with a variety of biocompatible ligands such as citrate, lysine and cysteine. The primary particles form clusters upon addition of a biodegradable polymer, PLA(1k)-bPEG(10k)-b-PLA(1k), followed by controlled solvent evaporation. The cluster size may be tuned from 20-40 nm in diameter by manipulating the gold and polymer concentrations along with the solvent evaporation extent. Salt is also added to increase the NIR absorbance and reduce the nanocluster size by reducing polymer adsorption. The adsorption of the polymer onto the Au surfaces effectively quenches the nanoclusters. High NIR absorption facilitates photoacoustic imaging, even for the small cluster sizes. In response to acidic cellular $\mathrm{pH}$ environments, the polymer degrades and the clusters dissociate back to primary particle on the order of $5 \mathrm{~nm}$, which are small enough for renal clearance.
\end{abstract}

Keywords: Gold nanoclusters, plasmonic, clearable, photoacoustic, biodegradable, biomedical imaging, protein adsorption

\subsection{INTRODUCTION}

Gold nanoparticles are of great interest as photoacoustic imaging contrast agents due to their ability to exhibit high extinction in the near infrared (NIR) wavelength range between $650-900 \mathrm{~nm}$ where tissue absorbs weakly. ${ }^{1-4}$ Such extinction can be achieved by minimizing interparticle spacings between particles which results in surface plasmon resonance (SPR) shifts due to the creation of hybridized plasmon modes between particles. ${ }^{5}$ High NIR extinction from gold nanoparticles have been observed in vitro via templated cluster assembly ${ }^{6-9}$ as well as in cells under high gold loadings. ${ }^{10,11}$ However such high-NIR active gold nanostructures are irreversible and thus remain non-clearable from the body due to their large size as particles with diameters less than $\sim 5.5 \mathrm{~nm}$ are required for efficient renal clearance. ${ }^{12,13} \mathrm{At}$ this size, the adsorption of even a single protein molecule during circulation could prevent efficient clearance. ${ }^{12,}$, 14-16, 9 Therefore, there is a need for closely-spaced gold nanoclusters with high NIR extinction which also completely dissociate back to $\sim 5 \mathrm{~nm}$ primary particles which lack protein adsorption to allow renal clearance.

Many attempts at making high NIR-active gold clusters via manipulation of electrostatic forces have yielded large and irreversible aggregates. ${ }^{17}, 18$ Greater size control has been shown via balancing steric and van der Waals forces between $\sim 12 \mathrm{~nm}$ poly(ethylene glycol) (PEG)-capped Au nanoparticles through the addition of hydrophobic alkane thiol molecules while altering the PEG surface concentration. ${ }^{19}$ Additionally, clusters of controlled size were assembled from 
$\sim 6 \mathrm{~nm}$ iron oxide nanoparticles via manipulation of solvophobic interactions between a hydrophilic ethylene glycol solvent and oleic acid ligands. ${ }^{20}$ However for both the gold and iron oxide particles, the clusters were irreversible.

Through a careful balance of the short-range attractive and long-range repulsive colloidal forces (equation 1), our group has developed a method for the assembly of biodegradable nanoclusters of controlled size. ${ }^{6-9}$

$$
V_{\text {Total }}=V_{v d W}+V_{\text {Electrostatic }}+V_{\text {Steric }}+V_{\text {Depletion }}
$$

A biodegradable polymer, PLA(1k)-PEG(10K)-PLA(1k), was used to "quench" the growth of the high-NIR active nanoclusters via adsorption of the hydrophobic PLA groups to the gold surface during the equilibrium formation mechanism. ${ }^{21,8}$ In our previous study, nanocluster sizes were tuned from $\sim 20 \mathrm{~nm}$ to $\sim 40 \mathrm{~nm}$ with varying degrees of NIR extinction. After formation, full dissociation of the clusters was demonstrated as the PLA anchoring groups underwent hydrolytic degradation in low $\mathrm{pH}$ environments.

Herein, we expand upon our previous designed clusters by including salt to vary degrees of polymer adsorption and thus alter interparticle spacings and NIR extinction. Two of our previously designed nanoparticle systems which have been shown to prevent protein adsorption are studied. ${ }^{9}$ Firstly, gold nanoparticles used in our previous equilibrium cluster formation study, capped with cationic lysine and anionic citrate are examined. A method to further decrease cluster size while maintaining high NIR extinction is desirable and is shown to be possible by limiting polymer adsorption within the cluster through salt addition. Secondly, Au nanoparticles capped with zwitterionic cysteine and citrate are of interest due to the strong binding affinity of the thiolated cysteine to gold. Due to the weak interaction of lysine with gold, ${ }^{22}$ a cysteine/citrate-capped gold surface would likely give increased ligand stability in vivo where intracellular thiol concentrations are high. ${ }^{23}$ Therefore we also report the formation of small, NIR-active clusters made from these nanoparticles in the presence of increasing ionic strength.

\subsection{MATERIALS \& METHODS}

\subsection{Materials}

Gold precursor, $\mathrm{HAuCl}_{4} \cdot 3 \mathrm{H}_{2} \mathrm{O}$ was purchased from Acros Chemicals (Morris Plains, NJ). Reducing agents $\mathrm{NaBH}_{4}$ and $\mathrm{Na}_{3} \mathrm{C}_{3} \mathrm{H}_{5} \mathrm{O}(\mathrm{COO})_{3} \cdot 2 \mathrm{H}_{2} \mathrm{O}$ were acquired from Fisher Scientific (Fair Lawn, NJ). Alternative ligands L-(+)Lysine and L-Cysteine were also purchased from Acros Chemicals while the triblock copolymer PLA(1k)-PEG(10k)PLA(1k) was attained from Sigma-Aldrich (St. Louis, MO). Pure fetal bovine serum (FBS) was acquired from Hyclone (Logan, UT).

\subsection{Synthesis of Citrate-capped Au nanospheres and cysteine/lysine ligand exchange}

Approximately $4 \mathrm{~nm}$ citrate-capped Au nanospheres were synthesized via $\mathrm{NaBH}_{4}$ reduction of $\mathrm{HAuCl}_{4}$ at $95{ }^{\circ} \mathrm{C}$ in the presence of $\mathrm{Na}_{3} \mathrm{C}_{3} \mathrm{H}_{5} \mathrm{O}(\mathrm{COO})_{3}$ and purified via tangential flow filtration and centrifugal filtration via a previously described procedure.(Murthy ACS Nano12) For the cysteine place exchange, $6.4 \mu 1$ of a freshly prepared $1 \%(\mathrm{w} / \mathrm{v})$ cysteine in deionized (DI) water solution was added to $1.2 \mathrm{~mL}$ of a $3 \mathrm{mg} / \mathrm{mL}$ citrate-capped Au nanosphere solution at room temperature. The mixture was stirred for 15 minutes, immediately after which the solution underwent characterization or cluster formation processes. The lysine place exchange was completed under the same reaction conditions but as a mixture of $20 \mu \mathrm{l}$ of a freshly prepared $5 \%(\mathrm{w} / \mathrm{v})$ lysine in DI water solution in $1.2 \mathrm{~mL}$ of a $3 \mathrm{mg} / \mathrm{mL}$ $\mathrm{Au}$ nanosphere dispersion. The resulting particles for both the cysteine and lysine ligand exchanges contain a 1.6/1 and $1.4 / 1$ ligand/citrate ratio, respectively as determined via x-ray photoelectron spectroscopy (XPS) as described in a previous study. ${ }^{8}$

\subsection{Au nanosphere characterization and protein adsorption test}

Nanosphere size, extinction, and ligand ratios were measured via previously described methods. ${ }^{8}$ Weightaverage hydrodynamic diameters of the particles were acquired via dynamic light scattering (DLS) measurements of 
$\sim 0.04 \mathrm{mg} / \mathrm{mL}$ Au nanosphere solutions. A Brookhaven ZetaPALS analyzer at a detector angle of $90^{\circ}$ was used and the data was analyzed via the CONTIN method and Stokes-Einstein equations. Extinction data of the resulting nanoparticle solutions were measured using a Cary $3 \mathrm{E}$ UV-Vis-NIR spectrophotometer with a $1 \mathrm{~cm}$ path length cuvette. X-ray photoelectron spectroscopy (XPS) was done to determine ligand ratios on the Au nanosphere surface and done on a Kratos AXIS Ultra DLD spectrometer with a monochromatic Al X-ray source. Full methodology for this test has been reported by Murthy et al. ${ }^{8,9}$

After place exchange, protein adsorption characteristics were studied by diluting $\mathrm{Au}$ nanospheres to $\sim 0.03$ $\mathrm{mg} / \mathrm{mL}$ in $100 \% \mathrm{FBS}$ and incubating the solution in a $37{ }^{\circ} \mathrm{C}$ water bath. DLS measurements were taken on the resulting solutions after 48 hours.

\subsection{Nanocluster formation and characterization}

Following place exchange, nanocluster formation was done following a familiar procedure. ${ }^{8}$ Lysine/citrate nanoclusters were made holding final gold and polymer concentrations constant at $2 \mathrm{mg} / \mathrm{mL}$ and $40 \mathrm{mg} / \mathrm{mL}$, respectively. A 20/1 polymer/Au (w/w) ratio of PLA(1k)-PEG(10k)-PLA(1k):Au was added to a $3 \mathrm{mg} / \mathrm{mL}$ solution of $1.4 / 1$ lysine/citrate $\mathrm{Au}$ nanospheres under stirring. The polymer solution contained between $50 \mathrm{mM}$ and $100 \mathrm{mM} \mathrm{NaCl}$ concentrations for desired final salinity. After polymer addition, $50 \%$ of the solvent was evaporated under dry air at room temperature

For cysteine/citrate nanoclusters, a 5/1 polymer/Au (w/w) ratio of PLA(1k)-PEG(10k)-PLA(1k):Au was added to a $3 \mathrm{mg} / \mathrm{mL}$ stirred solution of $1.6 / 1$ cysteine/citrate Au nanospheres. The polymer was added in five equal iterations over 10 minutes at room temperature. As for the lysine/citrate clusters the $30 \mathrm{mg} / \mathrm{mL}$ polymer solution contained a 100 $\mathrm{mM} \mathrm{NaCl}$ concentration. After polymer addition was complete, samples underwent $50 \%$ and $95 \%$ solvent evaporation at room temperature under a dried air flow.

For both systems, nanocluster formation was quenched by diluting the samples to $\sim 30 \mathrm{~mL}$ with DI water in 50 $\mathrm{mL}$ centrifuge tubes. The samples were bath sonicated and then centrifuged at 10,000 rpm for 10 minutes to isolate a pellet of densely-packed nanoclusters from the supernatant which contained unreacted primary nanospheres and small, loosely-packed aggregates. DLS and UV-VIS-NIR measurements were then taken of pellet. ${ }^{8}$

\subsection{Nanocluster dissociation}

In vitro dissociation tests were done on the 2-40-67-50 lysine and citrate and 40-200-668-95 cysteine and citrate nanoclusters by dispersing $200 \mu \mathrm{L}$ of a $\sim 2 \mathrm{mg} / \mathrm{mL}$ respective nanocluster solution in $2.4 \mathrm{~mL}$ of a $\mathrm{pH} 5 \mathrm{HCl}$ solution. The mixtures were incubated in a $37^{\circ} \mathrm{C}$ water bath over a 24 hour period throughout which extinction and hydrodynamic

diameter measurements were taken periodically via UV-Vis-NIR and DLS analysis at similar conditions as previously described.

\subsection{RESULTS \& DISCUSSION}

\subsection{Lysine/Citrate Nanoclusters}

Lysine and citrate capped $\mathrm{Au}$ nanoparticles were assembled into clusters at two final $\mathrm{NaCl}$ concentrations, $67 \mathrm{mM}$ and $133 \mathrm{mM}$ while the final gold and polymer concentrations were held at $2 \mathrm{mg} / \mathrm{mL}$ and $40 \mathrm{mg} / \mathrm{mL}$, respectively. Figure 1a shows significant NIR extinction was achieved for both concentrations of $\mathrm{NaCl}$ compared to unclustered primary particles. Meanwhile, a noticeable size increase from $23.7 \mathrm{~nm}(2-40-67-50)$ to $35.6 \mathrm{~nm}$ (2-40-133-50) was observed as the $\mathrm{NaCl}$ concentration was increased as shown in figure $1 \mathrm{~b}$. Since increased salinity is known to lower electrostatic repulsion via ion pairing, this effect likely results in the further aggregation of nanoparticles into larger clusters. However despite this size increase, little difference was observed in extinction from $67 \mathrm{mM}$ to $133 \mathrm{mM} \mathrm{NaCl}$, indicating a negligible effect of increasing cluster diameter on NIR extinction in this size range for these conditions. 

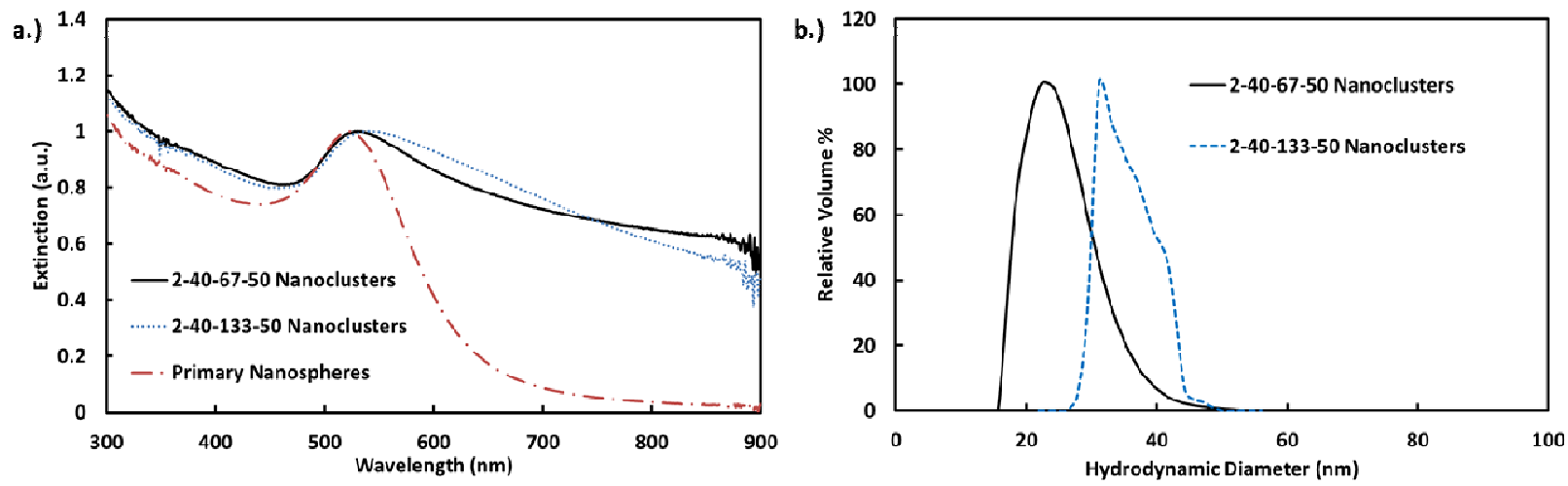

Figure 1: a.) UV-Vis and b.) DLS data for $2 \mathrm{mg} / \mathrm{mL}$ final $\mathrm{Au}$ and $40 \mathrm{mg} / \mathrm{mL}$ final polymer lysine/citrate nanoclusters at varying final $\mathrm{NaCl}$ concentrations

After formation, full dissociation of the 20-50-67-50 lysine/citrate nanoclusters was demonstrated by incubating them in a pH $5 \mathrm{HCl}$ solution at $37{ }^{\circ} \mathrm{C}$ for 24 hours to imitate intercellular conditions. During incubation, the PLA chains on the triblock copolymer adsorbed to the clusters undergoes rapid hydrolysis, removing the cluster stabilizing agent. Once removed, the residual charge on the primary particles provides enough electrostatic repulsion to push the particles back to primary constituent form. The UV-Vis and DLS analysis in figure 2 shows the full dissociation of the clusters. The UV-Vis shows the extinction curve of the clusters going nearly all the way back to that of primary nanospheres whereas the DLS shows the diameter dropping to the original $\sim 5 \mathrm{~nm}$ primary particle size after incubation. The resulting primary particles remained stable in this solution for weeks.
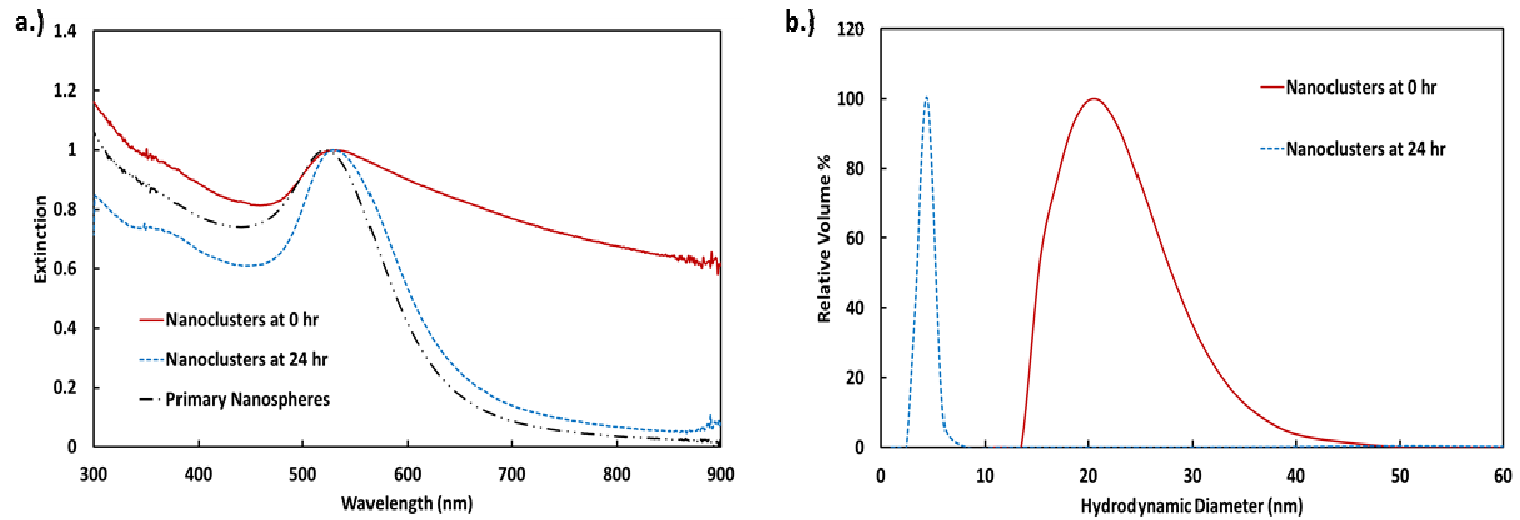

Figure 2: a.) UV-Vis and b.) DLS curves showing full dissociation of 2-40-67-50 lysine/citrate nanoclusters after 24 hour incubation in $\mathrm{pH} 5 \mathrm{HCl}$ solution

\subsection{Cysteine/Citrate Nanoclusters}

Similar to the lysine/citrate nanoclusters, primary nanospheres of cysteine/citrate were assembled into clusters of controlled size with the addition of salt. Using an initial gold concentration of $3 \mathrm{mg} / \mathrm{mL}$ and a polymer:Au ratio of 5:1 $(\mathrm{w} / \mathrm{w})$, clusters were made in the presence of $\mathrm{NaCl}$ at varying solvent evaporation extents. Figure $3 \mathrm{a}$ shows that as solvent evaporation was increased to $95 \%$ (40-200-668-95) from 50\% (4-20-67-50), a significant increase in NIR extinction was observed. Since solvent evaporation simultaneously increases gold, polymer and $\mathrm{NaCl}$ concentrations, both vdW and depletion attraction were increased while electrostatic repulsion decreased due to ion pairing. Therefore the subsequent increase in interparticle interactions likely resulted in closely-packed aggregates and the improved NIR extinction at high evaporation extents. 
a.)

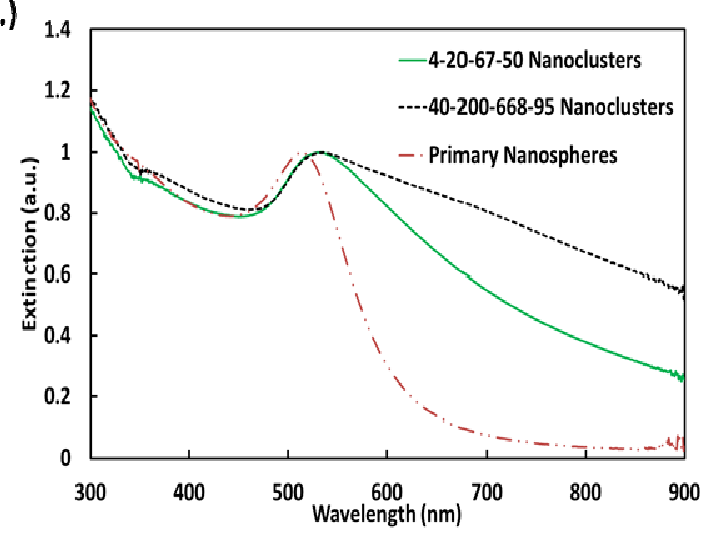

b.)

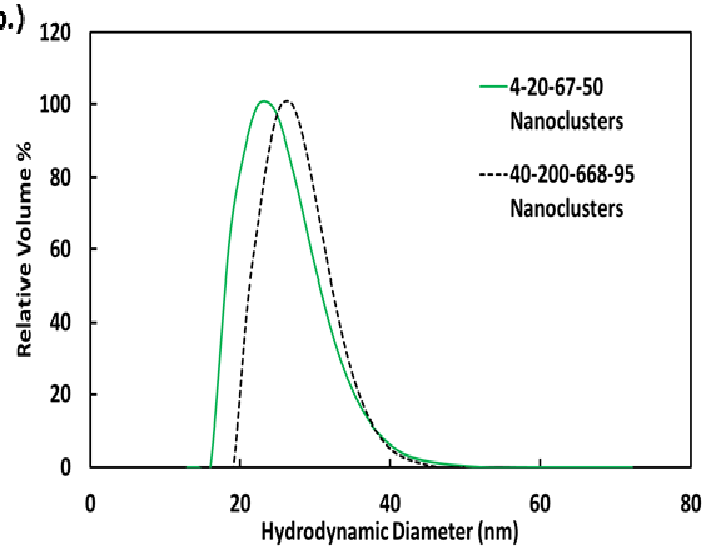

Figure 3: a.) UV-Vis and b.) DLS data for cysteine/citrate nanoclusters at $50 \%$ and $95 \%$ evaporation made in presence of $\mathrm{NaCl}$

Interestingly, despite the higher NIR extinction, the nanoclusters only increased in size from $\sim 25 \mathrm{~nm}$ to $\sim 27 \mathrm{~nm}$ as the evaporation extent increased from $50 \%$ to $95 \%$ (figure $3 \mathrm{~b}$ ). It is possible the increased solution concentration of gold, polymer, and salt resulted in the formation of more dense nanoclusters at $95 \%$ evaporation which would explain the higher NIR extinction without a significant size increase.

No nanocluster dissociation data are presented for cysteine/citrate nanoclusters as they were unstable in pH 5 $\mathrm{HCl}$ after overnight incubation. Further studies are underway to understand and overcome this limitation.

\subsection{Particle Protein Adsorption}

Following incubation of lysine/citrate and cysteine/citrate primary nanospheres in 100\% FBS for 48 hours at 37 ${ }^{\circ} \mathrm{C}$, no protein adsorption was detected as no increase in hydrodynamic diameter was shown through DLS analysis of the samples. Since the primary constituent of FBS, bovine serum albumin (BSA), is $\sim 3.5 \mathrm{~nm}$ in diameter, even a single protein would increase the hydrodynamic diameter. However as seen in figure 4, only citrate-capped gold nanospheres show a significant size increase indicative of protein adsorption.

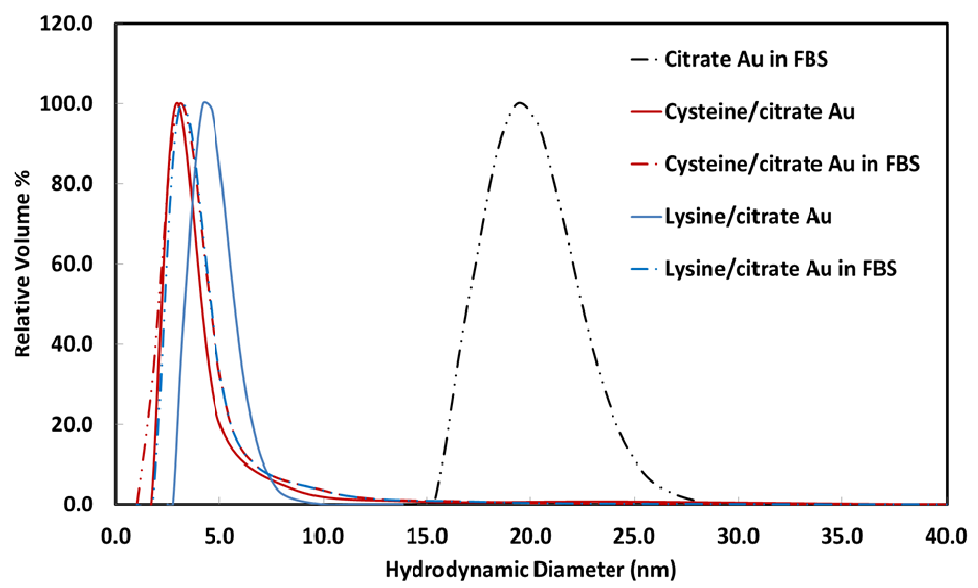

Figure 4: DLS data for lysine/citrate and cysteine/citrate and citrate-only capped Au nanospheres before and after 48 hour incubation in $100 \% \mathrm{FBS}$

Previous literature has shown that highly charged gold surfaces show increased protein adsorption due to their interaction with charged domains on protein molecules. ${ }^{24}$ However, for neutral, mixed-charge or zwitterionic surfaces, little protein adsorption is seen likely due to lower charge interactions and the presence of hydration layers around such surfaces. ${ }^{24-26}$ 


\section{CONCLUSIONS}

The ability to assemble high NIR-active gold nanoclusters of controlled size, quenched with biodegradable polymer stabilizers has been shown for two dual-ligand systems. Through the manipulation of gold, polymer, and salt concentration, the size and spectra of lysine/citrate and cysteine/citrate nanoclusters have been effectively tailored to produce high NIR extinction from small, $<30 \mathrm{~nm}$ clusters, adequate for use as photoacoustic contrast agents. The clusters were assembled from primary nanospheres which adsorb no protein molecules after incubation in $100 \%$ FBS and full dissociation of lysine/citrate nanoclusters has been demonstrated in conditions similar to intracellular environments producing nanospheres with the $<5 \mathrm{~nm}$ size required for efficient renal clearance.

\section{ACKNOWLEDGEMENTS}

This research was supported by NSF grant CBET-0968038 and NIH grant CA143663, along with the Welch Foundation (F-1319).

\section{REFERENCES}

[1] Link, S.; Mohamed, M. B.and El-Sayed, M. A., "Simulation of the Optical Absorption Spectra of Gold Nanorods as a Function of Their Aspect Ratio and the Effect of the Medium Dielectric Constant," J. Phys. Chem. B 103(16), 3073-3077 (1999).

[2] Weissleder, R., "A clearer vision for in vivo imaging," Nat. Biotechnol. 19/(2001).

[3] Mallidi, S.; Larson, T.; Tam, J.; Joshi, P. P.; Karplouk, A.; Sokolov, K.and Emelianov, S., "Multiwavelength Photoacoustic Imaging and Plasmon Resonance Coupling of Gold Nanoparticles for Selective Detection of Cancer," Nano Lett. 9(8), 2825-2831 (2009).

[4] Yoon, S. J.; Mallidi, S.; Tam, J. M.; Tam, J. O.; Murthy, A.; Johnston, K. P.; Sokolov, K. V.and Emelianov, S. Y., "Utility of biodegradable plasmonic nanoclusters in photoacoustic imaging," Opt. Lett. 35(22), 3751-3753 (2010).

[5] Halas, N. J.; Lal, S.; Chang, W.-S.; Link, S.and Nordlander, P., "Plasmons in Strongly Coupled Metallic Nanostructures," Chem. Rev. 111(3913-3961 (2011).

[6] Tam, J. M.; Murthy, A. K.; Ingram, D. R.; Nguyen, R.; Sokolov, K. V.and Johnston, K. P., "Kinetic Assembly of Near-IR Active Gold Nanoclusters Using Weakly Adsorbing Polymers to Control the Size," Langmuir 26(11), 8988-8999 (2010).

[7] Tam, J. M.; Tam, J. O.; Murthy, A.; Ingram, D. R.; Ma, L. L.; Travis, K.; Johnston, K. P.and Sokolov, K. V., "Controlled Assembly of Biodegradable Plasmonic Nanoclusters for Near-Infrared Imaging and Therapeutic Applications," ACS Nano 4(4), 2178-2184 (2010).

[8] Murthy, A. K.; Stover, R. J.; Borwankar, A. U.; Nie, G. D.; Gourisankar, S.; Truskett, T. M.; Sokolov, K. V.and Johnston, K. P., "Equilibrium Gold Nanoclusters Quenched with Biodegradable Polymers," ACS Nano 7(1), 239-251 (2013).

[9] Murthy, A. K.; Stover, R. J.; Hardin, W. G.; Schramm, R.; Nie, G. D.; Gourisankar, S.; Truskett, T. M.; Sokolov, K. V.and Johnston, K. P., "Charged Gold Nanoparticles with Essentially Zero Serum Protein Adsorption in Undiluted Fetal Bovine Serum," J. Am. Chem. Soc. 135(21), 7799-7802 (2013). [10] Aaron, J.; Nitin, N.; Travis, K.; Kumar, S.; Collier, T.; Park, S. Y.; Jose-Yacaman, M.; Coghlan, L.; Follen, M.; Richards-Kortum, R., et al., "Plasmon resonance coupling of metal nanoparticles for molecular imaging of carcinogenesis in vivo," Journal of Biomedical Optics 12(3), (2007). 
[11] Aaron, J.; Travis, K.; Harrison, N.and Sokolov, K., "Dynamic Imaging of Molecular Assemblies in Live Cells Based on Nanoparticle Plasmon Resonance Coupling," Nano Lett. 9(10), 3612-3618 (2009).

[12] Choi, H. S.; Liu, W.; Misra, P.; Tanaka, E.; Zimmer, J. P.; Ipe, B. I.; Bawendi, M. G.and Frangioni, J. V., "Renal clearance of quantum dots," Nat. Biotechnol. 25(10), 1165-1170 (2007).

[13] Zhou, C.; Long, M.; Qin, Y.; Sun, X.and Zheng, J., "Luminescent Gold Nanoparticles with Efficient Renal Clearance," Angew. Chem. Int. Ed. 50(3168-3172 (2011).

[14] Ladd, J.; Zhang, Z.; Chen, S.; Hower, J. C.and Jiang, S., "Zwitterionic Polymers Exhibiting High Resistance to Nonspecific Protein Adsorption from Human Serum and Plasma," Biomacromolecules 9(5), 1357-1361 (2008).

[15] Yang, W.; Xue, H.; Li, W.; Zhang, J.and Jiang, S., "Pursuing "Zero" Protein Adsorption of Poly(carboxybetaine) from Undiluted Blood Serum and Plasma," Langmuir 25(19), 11911-11916 (2009).

[16] Yang, W.; Zhang, L.; Wang, S.; White, A. D.and Jiang, S., "Functionalizable and ultra stable nanoparticles coated with zwitterionic poly(carboxybetaine) in undiluted blood serum," Biomaterials 30(5617-5621 (2009). [17] Wilcoxon, J. P.; Martin, J. E.and Schaefer, D. W., "Aggregation in colloidal gold," Physical Review A 39(5), 2675-2688 (1989).

[18] Chow, M. K.and Zukoski, C. F., "Gold Sol Formation Mechanisms: Role of Colloidal Stability," J. Colloid Interface Sci. 165(97-109 (1994).

[19] Larson-Smith, K.and Pozzo, D. C., "Scalable synthesis of self-assembling nanoparticle clusters based on controlled steric interactions," Soft Matter 7/5339-5347 (2011).

[20] Zhuang, J.; Wu, H.; Yang, Y.and Cao, Y. C., "Controlling Colloidal Superparticle Growth Through Solvophobic Interactions," Angew. Chem. Int. Ed. 47(2208-2212 (2008).

[21] Groenewold, J.and Kegel, W. K., "Anomalously Large Equilibrium Clusters of Colloids," J. Phys. Chem. B 105(11702-11709 (2001).

[22] Joshi, H.; Shirude, P. S.; Bansal, V.; Ganesh, K. N.and Sastry, M., "Isothermal Titration Calorimetry Studies on the Binding of Amino Acids to Gold Nanoparticles," J. Phys. Chem. B 108(31), 11535-11540 (2004).

[23] Verma, A.; Simard, J. M.; Worrall, J. W. E.and Rotello, V. M., "Tunable Reactivation of NanoparticleInhibited $\beta$-Galactosidase by Glutathione at Intracellular Concentrations," J. Am. Chem. Soc. 126(43), 1398713991 (2004).

[24] Holmlin, R. E.; Chen, X.; Chapman, R. G.; Takayama, S.and Whitesides, G. M., "Zwitterionic SAMs that Resist Nonspecific Adsorption of Protein from Aqueous Buffer," Langmuir 17(9), 2841-2850 (2001).

[25] Chen, S.; Zheng, J.; Li, L.and Jiang, S., "Strong Resistance of Phosphorylcholine Self-Assembled Monolayers to Protein Adsorption: Insights into Nonfouling Properties of Zwitterionic Materials," J. Am. Chem. Soc. 127(41), 14473-14478 (2005).

[26] Chen, S.; Yu, F.; Yu, Q.; He, Y.and Jiang, S., "Strong Resistance of a Thin Crystalline Layer of Balanced Charged Groups to Protein Adsorption," Langmuir 22(19), 8186-8191 (2006). 